\title{
UNA NUEVA ESPECIE TROPICAL DE POPULUS (SALICACEAE) DE LA SIERRA DE MANANTLAN, JALISCO, MEXICO'
}

\author{
Antonio Vazquez y Ramon Cuevas \\ Laboratorio Natural Las Joyas \\ Universidad de Guadalajara \\ 48740 El Grullo, Jal., México
}

\begin{abstract}
RESUMEN
Se describe una nueva especie de Populus de la Sierra de Manantlán, Jalisco, México: Populus guzmanantlensis (secc. Populus, subsecc. Tomentosae). Las especies con las que parece tener mayor parentesco son $P$. simaroa Rzedowski y $P$. monticola T.S. Brandegee, pero se distingue de ambas claramente por su mayor número de flores en los amentos femeninos, y por sus frutos pequeños y glabros.
\end{abstract}

\begin{abstract}
A new species of Populus is described from the Sierra of Manantlán, state of Jalisco, México: Populus guzmanantlensis (sect. Populus, subsect. Tomentosae). It seems to be closely related to $P$. simaroa Rzedowski and $P$. monticola T.S. Brandegee, but is clearly different because of its larger number of flowers in the female aments and its smaller and glabrous fruits.

A finales de 1986 se colectaron en la Sierra de Manantlán, Jalisco, las últimas hojas y frutos de un árbol, que por la forma y la textura coriácea de sus hojas y sus dientes terminados en glándulas se identificó como perteneciente al género Populus. Fue hasta finales de 1988, cuando después de haber colectado bastantes hojas, flores y frutos, conseguido la mayor cantidad de literatura sobre el género y al no poderlo identificar, se concluyó que se trataba de una nueva especie.
\end{abstract}

\section{Populus guzmanantlensis sp. nov.}

Arbor usque ad $45 \mathrm{~m}$ alta, ramorum et truncorum juniorum cortex albus, levis, truncorum cortex senex longitudinaliter sulcatus; ramuli juniores dense pilosi usque ad sericei; gemmae dense albo-sericeae, parum viscosae, parum resinosae. Petioli (2) 4-8 (10) cm longi, plus minusve lateraliter compressi; lamina plerumque ovata vel orbicularis, apice acuta vel acuminata usque ad obtusa, basi plerumque rotundata vel obtusa usque ad cordata, margine irregulariter crenata vel serrata, firma coriacea, supra intense viridis et glabra vel fere flabra, subtus pallide viridis, plerumque tomentosa, saepe palmatinervata, nervi straminei. Inflorescentiae masculae usque ad $3 \mathrm{~cm}$ longae, densiflorae; bracteolae $3 \mathrm{~mm}$ longae, integrae, laciniatae; pedicelli 1-1.5 mm longi; discus $1 \mathrm{~mm}$ diametro, horizontalis vel meniscoideus; stamina 715 , antherae late oblongae. Inflorescentiae femineae densiflorae, 1-1.7 cm longae; bracteolae

'Trabajo realizado con apoyo del CONACYT, del Gobierno del Estado de Jalisco, del Fondo Mundial de la Vida Silvestre (W. W. F.) y de la Universidad de Wisconsin. 
$1.5 \mathrm{~mm}$ longae, integrae, laciniatae; pedicelli $0.6 \mathrm{~mm}$ longi; discus cupulatus; ovarium conicum, glabrum, uniloculare, placentis 2 basalibus et parietalibus, utraque 3 ovulis; styli 2, uterque 2 stigmata ferens. Capsula ovoideo-conica, 2-2.5 mm longa, 1-2 mm diametro, glabra, valvae 2, curvatae. Semina plerumque 4-6, $0.3 \mathrm{~mm}$ longa.

Arbol dioico hasta de $45 \mathrm{~m}$ de altura, tronco de 0.5 a $1.9 \mathrm{~m}$ de diámetro; copa densa y oblonga; fuste comúnmente limpio, ramificándose en su mitad superior; corteza delgada, lisa y blanquecina en los troncos jóvenes, tornándose gruesa, oscura y longitudinalmlente surcada en los adultos; ramas inferiores horizontales y en aparente verticilo, las superiores y medias ascendentes y alternas; ramillas tiernas densamente tomentosas, verde-amarillentas, con pelos unicelulares de 0.4-0.8 mm de largo, tornándose glabras y café rojizas en la madurez; lenticelas conspicuas, oblongas a elípticas; yemas ovado-cónicas, comunmente de 3 a $5 \mathrm{~mm}$ de largo por 2 a $3 \mathrm{~mm}$ de ancho, densamente blanco-tomentosas al principio, posteriormente glabras y rojizas, levemente resinosas y viscosas con las escamas caedizas.

Peciolos de (2) 4-8 (10) cm de largo, cilíndricos o levemente comprimidos lateralmente, densamente tomentosos al principio a glabrescentes o glabros en la madurez; limbo ovado a orbicular, en ocasiones romboideo, de 6-12 (16) cm de largo, de 5-11 (13) cm de ancho; ápice agudo, acuminado, obtuso o redondeado; base redondeada, obtusa o cordada, rara vez oblicua; margen irregularmente crenado, serrado o dentado, con la base generalmente subentera, con 2 a 3 dientes glandulares por centímetro y con 0 a 3 glándulas en la base de la lámina, orbiculares a oblongas. Hojas expuestas a la sombra papiráceas y de mayor tamaño, las expuestas al sol pequeñas y coriáceas; haz cubierto de una cutícula cerosa, glabro o glabrescente, de color verde intenso, con 3-5 nervaduras amarillentas principales; envés densamente tomentoso, las nervaduras prominentes y amarillas, las del último orden dando lugar a los dientes terminados en una glándula orbicular y rojiza (Fig. 1).

Inflorescencias masculinas cilíndricas, densifloras, de 1-3 cm de longitud, en ocasiones con el ápice levemente encorvado, raquis hasta de $1 \mathrm{~mm}$ de grueso, rojizo; bracteolas espatuladas de 2-3 mm de largo, margen dentado a partido, ciliadas, café claras a rojizas, con pubescencia caediza; flores estaminadas pediceladas, de 1-1.5 mm de longitud; disco floral plano a meniscoideo, de 0.7-1 mm de diámetro, amarillento; estambres en número de 7 a 15 , libres, filamentos glabros de 0.5-0.6 mm de largo, anteras basifijas y anchamente oblongas de 0.6-0.8 mm de longitud, amarillas.

Inflorescencias femeninas cilíndricas, densifloras, flexibles y levemente encorvadas, de 1-1.7 cm de largo, con 70-130 flores, volviéndose rígidas y hasta de $8 \mathrm{~cm}$ de largo en la fructificación; raquis en su parte central de más o menos $1 \mathrm{~mm}$ de diámetro, moreno-rojizo y glabro; bracteolas espatuladas u ovadas de 1.25-1.5 mm de longitud, de 0.75-1 $\mathrm{mm}$ de ancho, margen dentado a lacerado, ciliado, con 3 a 4 dientes, levemente estriadas, caedizas y glabras, café-amarillentas a rojizas; flores femeninas de 1.5-2 mm de largo; pedicelos de 0.5-0.6 mm de longitud, glabros; disco floral en forma de copa, con la porción abaxial más pronunciada, persistente y glabro; ovario cónico, bicarpelar, unilocular con placentación basal parietal, placentas 2 con 3 óvulos en cada una; estilo doblemente bifurcado.

Cápsulas ovoideo-cónicas de (1.75) 2-2.5 (3) mm de longitud, de 1-2 mm de diámetro, superficie papilosa y glabra, dehiscencia apical, abriéndose por suturas ventrales en 2 valvas revolutas.

Semillas 4 a 6 por cápsula, elípticas a oblongas, de $0.3 \mathrm{~mm}$ de largo, glabras, amarillentas a rojizas. Pelos del interior de la cápsula de color blanco a amarillentos (Fig. 2). 
Vázquez y Cuevas: Una Nueva Especie de Populus

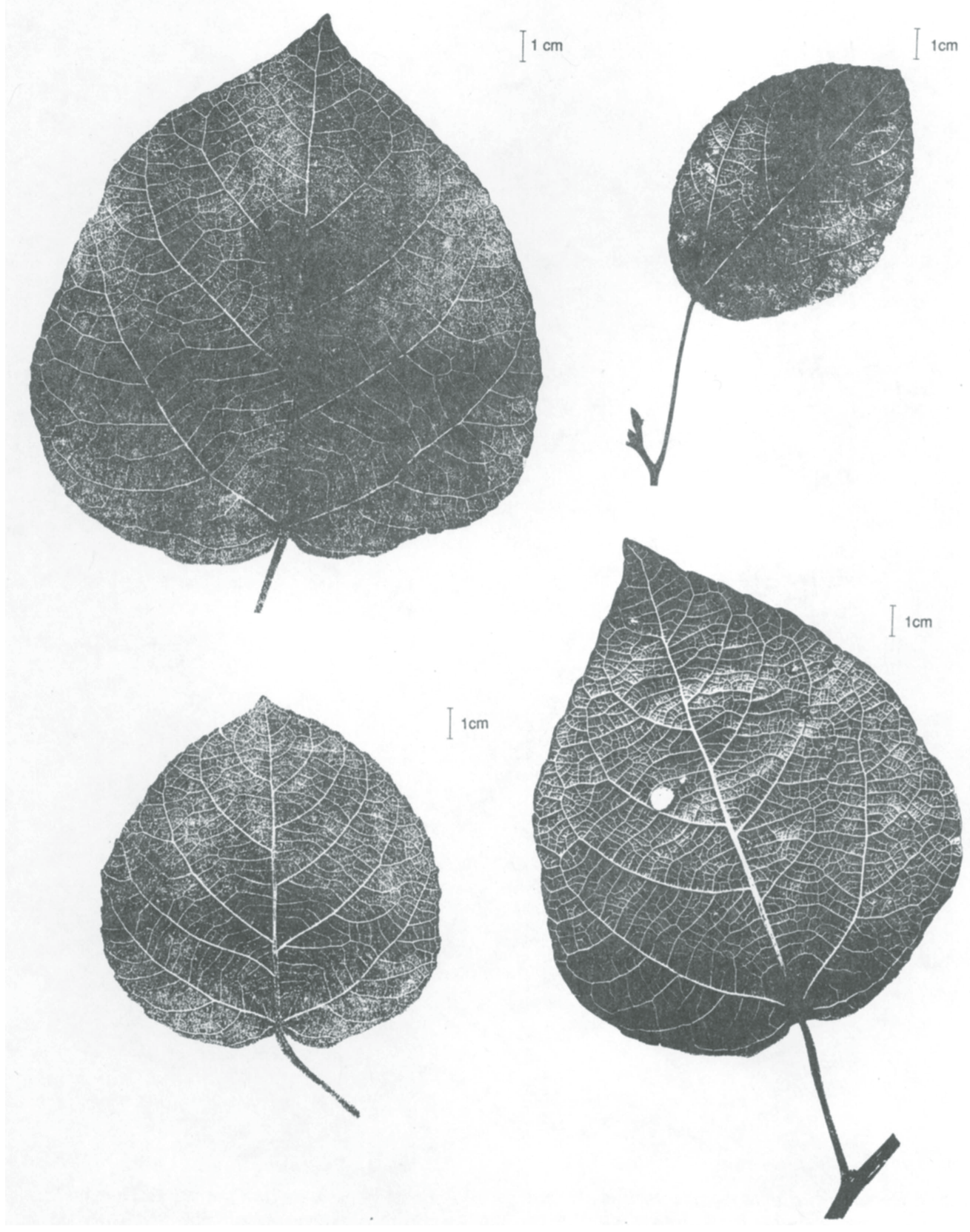

Fig. 1. Variabilidad de la hoja (Iltis et al. 30101). 

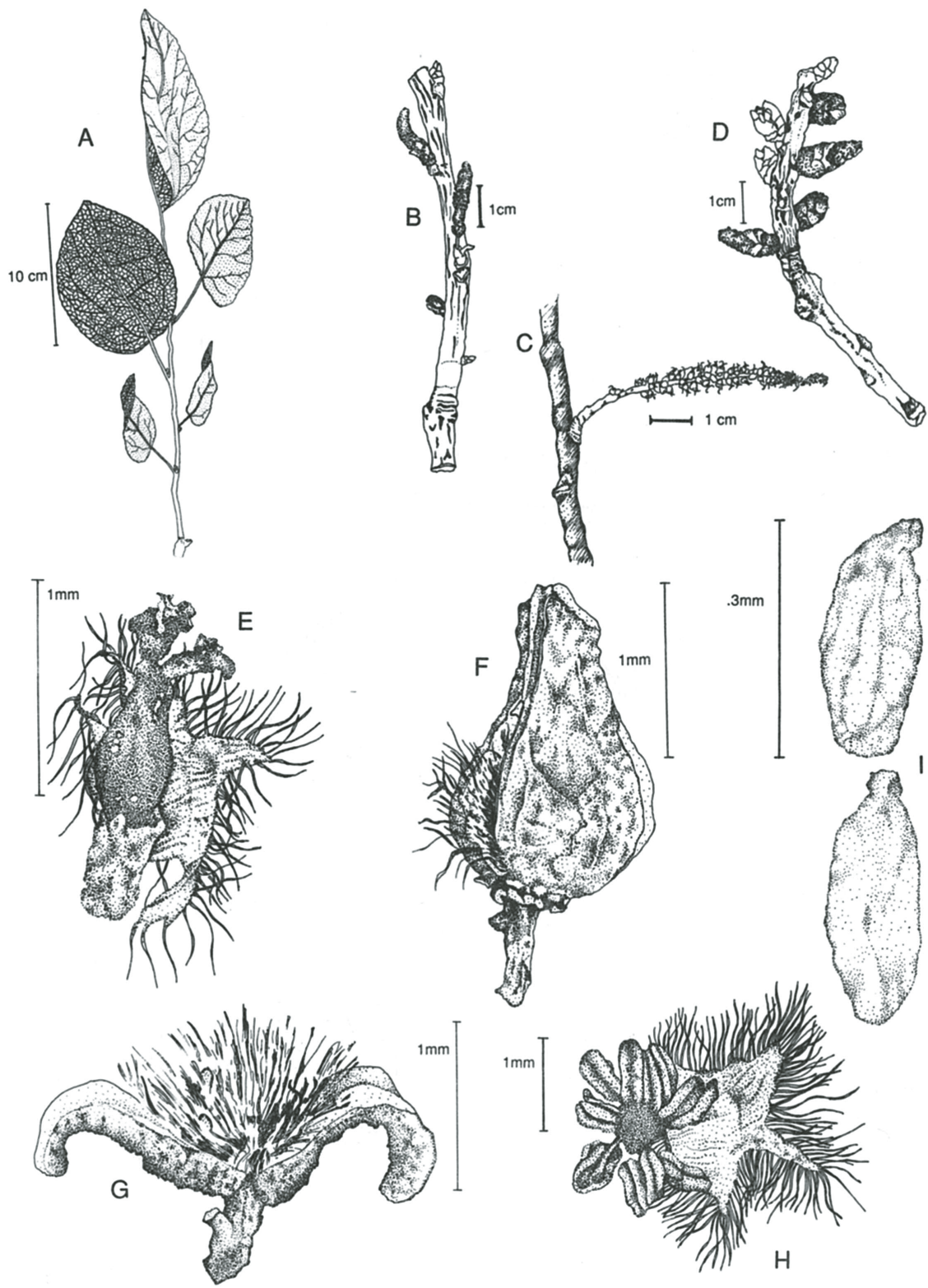

Fig. 2. Populus guzmanantlensis Vázquez \& Cuevas. A. Aspecto general de una ramilla; B. Ramilla con inflorescencias femeninas; C. Infrutescencia; D. Ramilla con inflorescencias masculinas; E. Pistilo y bracteola; F. Cápsula mostrando su aspecto general; G. Cápsula abierta; H. Estambres y bracteola; I. Semillas. (R. Cuevas y L. Guzmán 3224; inflorescencia masculina de 3224a). 
Florece y fructifica de (septiembre) octubre a noviembre, defoliándose de agosto a octubre (noviembre).

TIPO: México, Jalisco, Sierra de Manantlán, municipio de Casimiro Castillo, 1-2 km al NE de Casimiro Castillo, 400-500 m de altitud, en bosque tropical subcaducifolio, 14. X.1988, R. Cuevas y L. Guzmán 3224 "femenino" (holotipo: ZEA).

Material adicional examinado: México, Jalisco, Sierra de Manantlán, 1-2 km al NE Casimiro Castillo, municipio de Casimiro Castillo, bosque tropical subcaducifolio, alt. 450-500 m, 20.V.1988 (hojas), R. Cuevas y L. Guzmán 2931 (ZEA); Sierra de Manantlán, 5-6 km al NE Casimiro Castillo, municipio de Casimiro Castillo, bosque tropical subcaducifolio, alt $750 \mathrm{~m}$, 20.III.1988 (hojas), B. Benz et al. 1184 (ZEA); Sierra de Manantlán, 1.5-2 km al NE de Casimiro Catillo, municipio de Casimiro Castillo, bosque tropical subcaducifolio, alt. $450 \mathrm{~m}, 18$.VIII.1988 (inflorescencias masculinas), A. Vázquez et al. 4888 (ZEA); Sierra de Manantlán, 1-2 km al E del Rancho EI Tecolote, municipio de Casimiro Castillo, bosque tropical subcaducifolio, alt. 850 900 m, 21.V.1988 (hojas), R. Cuevas y L. Guzmán 2932 (ZEA); Sierra de Manantlán, 5-6 km al NE de Casimiro Castillo, municipio de Casimiro Castillo, bosque tropical subcaducifolio, alt. 600-850 m, 21. V. 1988 (hojas), R. Cuevas y L. Guzmán 2925 (ZEA); Sierra de Manantlán, 1$2 \mathrm{~km}$ al NE de Casimiro Castillo, municipio de Casimiro Castillo, bosque tropical subcaducifolio, alt. 450-500 m, 20.V.1988 (hojas), R. Cuevasy L. Guzmán.2930(ZEA); Siera de Manantlán, $1-3 \mathrm{~km}$ al NE de Casimiro Castillo, municipio de Casimiro Castillo, bosque tropical subcaducifolio, alt. 400-500 m, 14.X.1988 (flores masculinas), R. Cuevas y L. Guzmán 3224a (ZEA); Sierra de Manantlán, 1-2 km al NE de Casimiro Castillo, municipio de Casimiro Castillo, bosque tropical subcaducifolio, alt. 420-460 m, 15.XII.1988 (hojas), H. Iltis et al. 30101 (WIS); Sierra de Manantlán, $250 \mathrm{~m}$ al W de Chancol, municipio de Cuautitlán, bosque mesófilo de montaña, alt. 1100 m, 30.IX.1986 (hojas y flores), R. Cuevas 1708(ZEA); Sierra de Manantlán, 2-3 km al NNW de Telcruz, municipio de Cuautitlán, bosque mesófilo de montaña, alt. $1200 \mathrm{~m}, 19 . \mathrm{VII} .1987$ (hojas), A. Vázquez y $R$. López s.n. (ZEA); Sierra de Manantlán, entre Ayotitlán y Chancol, municipio de Cuautitlán, bosque tropical subcaducifolio, alt. $1000 \mathrm{~m}, 23.1 \mathrm{l} .1989$ (hojas), F. J. Santana et al. 4301 (ZEA); Sierra de Manantlán, $1 \mathrm{~km}$ al SE de la Pasión, municipio de Tuxcacuesco, bosque tropical subcaducifolio, alt. $1250 \mathrm{~m}, 6$. V.1988 (hojas y frutos residuales), R. Cuevas 2922 (ZEA).

Habitat: Se le encuentra confinado principalmente al bosque tropical subcaducifolio, pero en algunas ocasiones penetra en bosque mesófilo de montaña; siempre ligado a corrientes de agua, en altitudes de 400-1250 m.

Se le conoce en la zona con los nombres vulgares de "álamo", "alamillo" y "algodoncillo".

La especie se nombra en honor del ilustre botánico Rafael Guzmán, quien hizo realidad el sueño de proteger y conservar la maravillosa e intrigante Sierra de Manantlán, de donde esta planta es oriunda.

Por sus yemas rojizas, pubescentes y delicadamente resinosas, las hojas ovadas a orbiculares con el margen irregularmente aserrado, crenado o dentado, sus anteras cortas, anchas y no apiculadas, estambres 7 a 15, brácteas florales con el margen ciliado, disco floral persistente y en forma de copa, ovario bicarpelar y cápsula ovoide, Populus guzmanantlensis 
se ubica en la sección Populus (= Leuce Duby) (Eckenwalder, 1977b). Por el denso tomento que cubre las yemas, ramillas jóvenes y envés de sus hojas, así como su peciolo cilíndrico, puede colocarse dentro de la subsección Tomentosae (Eckenwalder, 1977a). Las especies con las que presenta mayor afinidad son $P$. monticola T.S. Brandegee y $P$. simaroa Rzedowski (Rzedowski, 1975); pero difiere de ambas en lo siguiente:

\begin{tabular}{|c|c|c|c|}
\hline Hojas & $\begin{array}{l}\text { P. monticola } \\
3-7 \times 2-4 \mathrm{~cm}\end{array}$ & $\begin{array}{l}\text { P. guzmanantlensis } \\
6-16 \times 5-13 \mathrm{~cm}\end{array}$ & $\begin{array}{l}\text { P. simaroa } \\
8-23 \times 4-13 \mathrm{~cm}\end{array}$ \\
\hline Inflorescencia & 25 a 60 flores & 70 a 130 flores & 25 a 75 flores \\
\hline Cápsulas & $\begin{array}{l}3.5 \text { a } 4 \mathrm{~mm} \text { de largo, } \\
\text { con pubescencia gris }\end{array}$ & $\begin{array}{l}2 \text { a } 3 \mathrm{~mm} \text { de largo, } \\
\text { glabras }\end{array}$ & $\begin{array}{l}4 \text { a } 6 \mathrm{~mm} \text { de largo, } \\
\text { con pubescencia gris }\end{array}$ \\
\hline Floración & diciembre a marzo & octubre a noviembre & marzo a junio \\
\hline Fructificación & junio a julio & octubre a noviembre & junio a julio \\
\hline Follaje & deciduo en invierno & deciduo en otoño & deciduo en verano \\
\hline Habitat & $\begin{array}{l}\text { praderas con mez- } \\
\text { quite y bosque de } \\
\text { encino }\end{array}$ & $\begin{array}{l}\text { bosque tropical sub- } \\
\text { caducifolio, bosque } \\
\text { mesófilo de montaña }\end{array}$ & $\begin{array}{l}\text { bosque mesófilo, bos- } \\
\text { que de pino y encino }\end{array}$ \\
\hline Altitud & 300 a $1700 \mathrm{~m}$ & 400 a $1250 \mathrm{~m}$ & 1500 a $2500 \mathrm{~m}$ \\
\hline $\begin{array}{l}\text { Distribución } \\
\text { geográfica }\end{array}$ & $\begin{array}{l}\text { Baja California Sur } \\
\text { y Sonora (Rzedowski, } \\
1985 \text { ) }\end{array}$ & $\begin{array}{l}\text { Jalisco (Sierra de } \\
\text { Manantlán) }\end{array}$ & $\begin{array}{l}\text { Estado de México y } \\
\text { Guerrero (Rzedowski, } \\
\text { 1985) }\end{array}$ \\
\hline
\end{tabular}

Populus guzmanantlensis prospera bastante bien en un habitat completamente tropical, llegando a tener un desarrollo exhuberante.

Su sistema de reproducción es por semillas, las cuales muestran alto procentaje de viabilidad; es poco usual el crecimiento de más de un tronco por individuo; las plántulas se establecen principalmente en los playones y pequeñas porciones de tierra dentro del cauce de los arroyos. Tal circunstancia parece ir en contra del desarrollo de las plántulas de la especie, debido a que la mayoría de ellas son arrastradas por fuertes corrientes de agua que se presentan durante los meses de julio a septiembre. Los individuos que se logran, llegan a tener un desarrollo exhuberante.

En cuanto a sus usos, se nos informó que la corteza es empleada como medicinal.

Urgen mayores estudios de esta especie, pues los atributos ya conocidos de este género, aunados a su adaptación extrema al calor, podrían resultar de enorme valor ecológico, económico y social, en el manejo de los bosques tropicales en la Sierra Madre del Sur u otros habitats similares en México.

\section{AGRADECIMIENTOS}

Los autores agradecen al Dr. H. Iltis, así como a R. Smith, B. Benz, T. Cochrane y K. Sytsma, de la Universidad de Wisconsin, por sus consejos y apoyo en la realización de este 
trabajo; al M.C. E. Jardel, director del Laboratorio Natural Las Joyas de la Universidad de Guadalajara, por las facilidades brindadas; al Dr. J. Rzedowski del Instituto de Ecología, por su gentileza en la revisión de este escrito; al Ing. Herminio Aceves y Don José Cruz por su ayuda en la búsqueda inicial de las poblaciones y al artista Manuel Reyes por la elaboración del dibujo.

\section{LITERATURA CITADA}

Eckenwalder, J.E. 1977a. Systematics of Populus L. (Salicaceae) in southwestern North America with special reference to sect. Aigeiros Duby. Tesis doctoral. University of California, Berkeley.

Eckenwalder, J.E. 1977b. North American cottonwoods (Populus, Salicaceae) of sections Abaso and Aigeiros. Journ. Arnold Arbor. 58(3): 193-208.

Rzedowski, J. 1975. Tres dicotiledoneas mexicanas nuevas de posible interés ornamental. Bol. Soc. Bot. Méx. 35:37-43.

Rzedowski, J. 1985. Análisis de la distribución geográfica de las especies mexicanas del género Populus (Salicaceae). C. R. Soc. Biogéog. (60)4: 138-140. 